\title{
Doenças Cardiovasculares Observadas durante o Seguimento de um Grupo de Pacientes na Forma Indeterminada da Doença de Chagas
}

\author{
Barbara Maria Ianni, Charles Mady, Edmundo Arteaga, Fábio Femandes
}

São Paulo, SP

\begin{abstract}
Objetivo - Observar, num grupo de pacientes na forma indeterminada da doença de Chagas, o aparecimento de doenças cardiovasculares e sua possível relação com a doença de base.

Métodos - Foram seguidos, prospectivamente, 160 pacientes por até 177 meses com avaliações clínicas trimestrais.

Resultados - Tornaram-se hipertensos 23 (14,4\%) pacientes, sendo 21 (13,2\%) com pressão arterial diastólica $<110 \mathrm{mmHg}$. Duas pacientes (1,2\%) hipertensas tiveram acidente vascular cerebral isquêmico (AVCI). Uma (0,6\%) paciente teve hemorragia meníngea por ruptura de aneurisma cerebral. Quatro $(2,4 \%)$ pacientes apresentaram arritmia clinicamente, dois $(1,2 \%)$ extrasistoles ventriculares, um $(0,6 \%)$ extra-sistoles supraventriculares e um $(0,6 \%)$ fibrilação atrial aguda. Dois $(1,2 \%)$ pacientes desenvolveram coronariopatia comprovada angiograficamente, um $(0,6 \%)$ com infarto agudo do miocárdio, outro com angina estável. Um $(0,6 \%)$ paciente desenvolveu sinais e sintomas de insuficiência cardiaca, juntamente com o aparecimento de hipertensão arterial sistêmica (HAS).

Conclusão - A doença cardiovascular mais freqüente foi a HAS. Duas hipertensas apresentaram AVCI. As arritmias observadas não foram mais freqüentes que na população normal e a coronariopatia também ocorreu raramente, confirmando um bom prognóstico clínico a longo prazo desse grupo de pacientes.
\end{abstract}

Palavras-chave: doença de Chagas, forma indeterminada, alterações clínicas

\section{Cardiovascular Diseases Observed During Follow-up of a Group of Patients in Undetermined Form of Chagas' Disease}

Purpose - The aim of this study was to evaluate the cardiovascular outcome in patients with the undetermined form of Chagas' disease and whether or not it is related to the infectious disease in the long-term.

Methods - One hundred and sixty patients were prospectively followed-up at three month intervals for up to 177 months.

Results - Twenty and three (14.4\%) patients developed hypertension complicated by ischemic stroke in two $(1.2 \%)$ and symptomatic heart failure in one (0.6\%). Cardiac arrhythmias occurred in four (2.4\%) patients corresponding to isolated ventricular ectopic beats in two $(1.2 \%)$, isolated supraventricular ectopic beats in one $(0.6 \%)$ and an isolated episode of acute atrial fibrillation in another (0.6\%). Two (1.2\%) patients developed symptoms of coronary artery disease, one of them had one episode of acute chest pain diagnosed as myocardial infarction and the other had chronic chest pain diagnosed as angina.

Conclusion - Hypertension is the most common cardiovascular disease occurring in the long-term followup of patients with the undetermined form of Chagas' disease. Cardiac rhythm disturbances and coronary artery disease were not more frequent than those generally found in a healthy population. These data confirm a favorable long-term prognosis in patients with the undetermined form of Chagas' disease.

Key-words: Chagas' disease, undetermined form, clinical changes

Arq Bras Cardiol, volume 71 (n 1), 21-24, 1998

Instituto do Coração do Hospital das Clínicas - FMUSP

Correspondência: Barbara Maria Ianni - Incor - Av. Dr. Enéas C. Aguiar, 44 - 05403 000 - São Paulo, SP

Recebido para publicação em 1/12/97

Aceito em 19/3/98
A doença de Chagas (DCh) caracteriza-se pela grande diversidade clínica, chegando porém, aos grandes centros de referência, apenas, os casos mais graves, cerca de $10 \%$ do número total de infectados no Brasil, estimado em cinco a seis milhões de pessoas ${ }^{1,2}$. Aproximadamente $60 \%$ dos 
chagásicos estão na forma indeterminada, isto é, são assintomáticos, têm reações sorológicas positivas para a doença, eletrocardiograma(ECG) eradiografia de tórax normais, além de estudo contrastado de esôfago e colo normais ${ }^{3-8}$. Essas pessoas frequientemente não chegam até o atendimento médico, ou quando o fazem é por outro motivo, já que comumente nem sabem que foram picadas pelo triatomídeo.

O objetivo do presente trabalho é descrever outras doenças cardiovasculares observadas durante a evolução de um grupo de pacientes na forma indeterminada, seguidos longitudinalmente, e discutir a possível relação entre elas e a infecção pelo Trypanosoma cruzi.

\section{Métodos}

Foram estudados 160 pacientes na forma indeterminada da DCh, sendo 98 mulheres e 62 homens, com idades entre $17 \mathrm{e}$ $61(36,5 \pm 8,8)$ anos, seguidos por 48 a $177(98,6 \pm 30,4)$ meses, com avaliações clínicas trimestrais, sendo o paciente inquerido sobre o aparecimento de sintomas comuns ao aparelho cardiovascular (principalmente dispnéia, dores torácicas, palpitações e edema de membros inferiores), ou outros quaisquer. Eram feitos exame clínico completo, com medida de pressão arterial, avaliação da freqüência cardíaca, auscultas pulmonar e cardíaca detalhadas, palpação de abdome para pesquisa de visceromegalia e exame de membros inferiores para avaliação de pulsos arteriais e pesquisa de edema.

A valorização da pressão arterial levou em conta o II Consenso Brasileiro de Hipertensão Arterial, que considera normal a pressão arterial diastólica (PAD) $<90 \mathrm{mmHg}$; entre 90 e $110 \mathrm{mmHg}$, as cifras são consideradas pouco aumentadas, podendo ser complicadas ou não, dependendo da existência de lesões em órgãos-alvo; a PAD $\geq 110 \mathrm{mmHg}$ é considerada como cifra alta, podendo também ser complicada ou não em relação ao comprometimento de órgãos-alvo ${ }^{9}$.

Os pacientes foram considerados na forma indeterminada quando tinham pelo menos duas reações sorológicas positivas para a doença (Guerrero-Machado e imunofluorescência), ECG, radiografia de tórax e estudo contrastado de esôfago e colo normais, além de serem assintomáticos em relação aos aparelhos cardiovascular e digestivo ${ }^{8,10,11}$. Todos os pacientes foram esclarecidos verbalmente sobre a programação do estudo e deram seu consentimento por escrito.

\section{Resultados}

Vinte e três $(14,4 \%)$ pacientes apresentaram alteração da PAD com valores $\geq 90 \mathrm{mmHg}$, sendo $21(13,2 \%)$ entre $90 \mathrm{e}$ $110 \mathrm{mmHg}$ e dois $(1,2 \%)$ com cifras superiores $(130 \mathrm{e}$ $140 \mathrm{mmHg})$. Duas $(1,2 \%)$ hipertensas tiveram acidente vascular cerebral isquêmico (AVCI) confirmado por tomografia computadorizada, com boa evolução. Nenhuma delas apresentava trombos intracavitários ou aneurisma de ponta ao ecocardiograma transtorácico. Em quatro $(2,4 \%)$ pacientes evidenciou-se arritmia clinicamente, sendo que, ao ECG, dois $(1,2 \%)$ tinham extra-sístoles ventriculares isoladas $(\mathrm{EV})$, um $(0,6 \%)$ extra-sístoles supraventriculares (ESV), e um $(0,6 \%)$ fibrilação atrial aguda $(\mathrm{FA})$, revertida quimicamente.

Um $(0,6 \%)$ paciente teve, após 104 meses de acompanhamento, infarto agudo do miocárdio (IAM) por obstrução total de artéria descendente anterior, confirmada pela cinecoronariografia. Outro $(0,6 \%)$ desenvolveu angina de peito típica aos grandes esforços, evoluindo com ECG de repouso normal e teste de esforço positivo. Realizada cinecoronariografia, ficou demonstrada obstrução total das artérias descendente anterior e coronária direita. Medicado, evoluiu assintomático.

Uma $(0,6 \%)$ paciente teve hemorragia meníngea por ruptura de aneurisma cerebral confirmado angiograficamente. Não era hipertensa e evoluiu sem seqüelas.

$\operatorname{Um}(0,6 \%)$ paciente desenvolveu sinais e sintomas de insuficiência cardíaca (ICC) após 108 meses de seguimento, coincidindo com o aparecimento de hipertensão arterial (diastólica em torno de $110 \mathrm{mmHg}$ ) e perda de ondas R em V1 e V2, além de aumento da cavidade ventricular esquerda (de 50 para 60mm) e diminuição da fração de ejeção de ventrículo esquerdo (de 0,68 para 0,55$)$.

\section{Discussão}

A alta prevalência de hipertensão arterial sistêmica (HAS) na população em geral leva a crer que numa população de pacientes chagásicos esta relação também existiria. O que se discute na literatura é se os mecanismos patogênicos envolvidos na DCh seriam também os responsáveis pela HAS nesse grupo de pessoas. Alterações de mecanismos adaptativos de barorreceptores em paciente na forma indeterminada já foram descritas ${ }^{12}$.

Trabalhos comparando pacientes chagásicos hipertensos com hipertensos não chagásicos demonstraram alta prevalência de HAS nos chagásicos, sendo $16,8 \%$ na forma indeterminada. Já nos pacientes com ICC, a presença de HAS chegava a 34\%, evidenciando caráter somatório e progressivo de ambas doenças ${ }^{13}$.

Em levantamento epidemiológico feito na Grande São Paulo foi observado que, no grupo de chagásicos, 26,6\% eram hipertensos, sendo aproximadamente $20 \%$ na forma indeterminada. Entre os não chagásicos, 20,3\% eram hipertensos, não havendo diferença estatisticamente significativa ${ }^{14}$. Porcentagem semelhante foi observada em zona endêmica $(20,99 \%){ }^{15}$. Na nossa casuística $23(14,4 \%)$ pacientes tornaram-se hipertensos durante o seguimento; a maioria, $21(13,2 \%)$ comPAD de, no máximo, $110 \mathrm{mmHg}$, números até inferiores aos referidos na literatura. Dos 23 pacientes, pudemos relacionar a HAS à presença de arritmia ventricular em um paciente e à presença de ICC em outro. Este paciente desenvolveu sintomas após os 60 anos de idade e seu quadro, segundo a experiência disponível na literatura, deve ter sido consequiência da associação entre a DCh, a HAS e a própria idade.

Outro aspecto clínico importante no seguimento dos pacientes foi a presença de acidentes vasculares cerebrais (AVC). Estudos de necropsia comparando chagásicos e não 
chagásicos não observaram diferença estatisticamente significativa em relação à presença de $\mathrm{AVC}$ nos dois grupos, sendo o AVCI mais freqüente nos chagásicos $(75 \%)$ em relação aos não chagásicos (31,3\%); o contrário foi observado em relação aos acidentes vasculares hemorrágicos (AVCH) (68,7\% nos não chagásicos e $25 \%$ nos chagásicos), nos quais a presença de HAS teve papel importante ${ }^{16}$. O mesmo foi observado com dados de tomografia computadorizada ${ }^{17}$. No nosso estudo, duas $(1,2 \%)$ pacientes hipertensas tiveram AVCI e uma $(0,6 \%)$ hemorragia meníngea por ruptura de aneurisma cerebral. É difícil explicar qual seria a etiologia mais provável dos AVCI, já que não havia trombo intraventricular nem aneurisma de ponta demonstrável ao ecocardiograma, apesar de não dispormos de ecocardiograma transesofágico dessas pacientes. Em relação à paciente com hemorragia meníngea, não havia HAS e apenas o aneurisma cerebral pode ser o responsabilizado.

Quanto às arritmias observadas, as EV tiveram porcentagem de aparecimento baixa (dois pacientes 1,2\%) sendo que em pessoas não chagásicas os números variam de $1 \%{ }^{18-21}$ a $54 \%{ }^{22-25}$, sendo os maiores de estudos com Holter. Além disso, a variabilidade da arritmia ventricular é um fato já descrito na $\mathrm{DCh}^{26-28}$. Quanto às ESV, a porcentagem de aparecimento nesse grupo (um caso 0,6\%) é também superponível à população normal, referida na literatura de $0,43 \%{ }^{6,18,29,30}$ a 5,8\% ${ }^{31}$. Em relação à $F A$, queé sempre relacionada à fase terminal da doença e referida como de mau prognóstico $^{32-35}$, não há referência na literatura relacionada ao seu aparecimento na evolução da forma indeterminada. De qualquer forma, no caso em questão, foi rapidamente revertida, sem haver comprometimento da função ventricular. Além disso, essa arritmia pode ser encontrada em pessoas normais de $0,06 \%$ a $1,88 \%{ }^{36}$. Portanto, as arritmias observadas não podem ser atribuídas à doença, já que a porcentagem de aparecimento é baixa e semelhante à população não chagásica.

Quanto à coronariopatia, existem evidências na literatura mostrando que IAM é causado por insuficiência coronária obstrutiva, tanto em chagásicos como em não chagásicos. Num estudo envolvendo 395 pacientes com IAM, 9,9\% deles chagásicos, coronárias normais foram encontradas em $7,7 \%$ dos chagásicos e em 7,8\% dos não chagásicos, não havendo diferenças também em relação às características clínicas, fatores de risco e evolução hospitalar nos dois grupos ${ }^{37}$. Em outro estudo, aterosclerose coronária esteve presente em $44 \%$ dos chagásicos e em $47 \%$ dos não chagásicos, não havendo também diferença estatisticamente significativa em relação à presença de $\mathrm{IAM}^{38}$. No nosso grupo, dois $(1,2 \%)$ pacientes tiveram clínica de insuficiência coronariana, um com angina de peito clássica, outro com IAM, sendo ambos portadores de coronariopatia obstrutiva, confirmada à cinecoronariografia. Apesar de não haver dados disponíveis na literatura em comparação com outros pacientes chagásicos na forma indeterminada, a insuficiência coronariana, como esperado pela observação clínica, é pouco freqüente.

Resumindo nosso estudo, a alteração clínica mais freqüente foi o aparecimento de HAS, seguida de arritmias ventricular e supraventricular e, menos freqüentemente, de AVCs e coronariopatia. Aparentemente não houve relação direta entre o aparecimento dessas alterações e a DCh em si, confirmando um bom prognóstico, a longo prazo, para os pacientes na forma indeterminada.

\section{Referências}

1. Dias JCP, Dias E - Doença de Chagas. In: Brasil. Ministério da Saúde - SUCAM. Doença de Chagas: texto de apoio. Brasília 1989; p.13-20.

2. Dias JCP - Profilaxia e impacto médico-social da doença de Chagas na região mineira do polígono das secas. Rev. Goiana Med 1982; 28: 97-102.

3. Chagas $\mathrm{C}$ - Processos patogênicos da tripanosomíase americana. Mem Inst Oswaldo Cruz: Rio de Janeiro, 1916; 8: 7-36.

4. Pereira-Barretto AC, Serro-Azul LG, Mady C et al - Forma indeterminada da doença de Chagas - uma doença polimórfica. Arq Bras Cardiol 1990; 55: 347-53.

5. Dias E, Laranja FS, Nóbrega G - Doença de Chagas. Mem Inst Oswaldo Cruz: Rio de Janeiro 1945; 42: 495-581.

6. Lopes ER, Chapadeiro E, Andrade ZA, Almeida HO, Rocha A - Anatomia patológica de corações de chagásicos assintomáticos falecidos de modo violento. Mem Inst Oswaldo Cruz 1981; 76: 189-97.

7. Macedo V - Inquérito eletrocardiográfico nacional para Doença de Chagas. Rev Soc Bras Med Trop 1993; 26: 12-3.

8. Primeira Reunião de Pesquisa Aplicada em Doença de Chagas. Validade do conceito da forma indeterminada. Rev Soc Bras Med Trop 1985 18: 46.

9. II Consenso Brasileiro de Hipertensão Arterial 1994. J Bras Nefrol 16 (Supl) 1994; 2: S259-S78.

10. Camargo ME, Oshino-Shimizu S, Macedo V, Peres BA, Castro C - Diagnóstico sorológico da infecção humana pelo Trypanosoma cruzi. Estudo comparativo de testes de fixação do complemento, imunofluorescência, hemaglutinação e floculação em 3.624 soros. Rev Inst Med Trop São Paulo 1977; 19: 254-60.

11. Mady C, Décourt LV - A forma indeterminada da doença de Chagas. Arq Bras Cardiol 1981; 36: 143-5.

12. Giorgi DMA, Lopes HF, Bernardes-Silva H, Pileggi F, Krieger EM - Depressed baroreflex sensitivity in hypertensive chagasic patients. Hypertension 1993; 21: 529 .
13. Guariento ME, Ramos MC, Gontijo JAR, Carvalhal SS - Doença de Chagas e hipertensão arterial primária. Arq Bras Cardiol 1993; 60: 71-5.

14. Medrado-Faria MA, Yasuda MAS, Araujo MJO, Lancarotte I, Capano EA, Ruiz Neto PP - Formas clínicas da doença de Chagas na Grande São Paulo. Arq Bras Cardiol 1982; 38: 99-109.

15. Pereira JB, Coura JR - Miocardite chagásica crônica: II - Influência da hipertensão arterial sistêmica. X Reunião Anual de Pesquisa Aplicada em Doença de Chagas, 1994: 72-3.

16. Lopes ER, Marquez JO, Costa Neto B, Menezes AAC, Chapadeiro E - Associação entre acidentes vasculares encefálicos e doença de Chagas. Rev Soc Bras Med Trop 1991; 24: 101-4.

17. Marquez JO, Blasczyk LC, Martins RMP, Lopes ER - Estudo dos acidentes vasculares encefálicos em chagásicos no Hospital Escola da Faculdade de Medicina do Triângulo Mineiro. X Reunião Anual de Pesquisa Aplicada em Doença de Chagas. Uberaba, 1994. Anais: 98-9.

18. Maguire JH, Hoff R, Sherlock I et al - Cardiac morbidity and mortality due to Chagas' disease: prospective electrocardiographic study of a Brazilian community. Circulation 1991; 45: 1140-5.

19. Maguire JH, Mott KE, Lehman JS et al - Relationship of electrocardiographic abnormalities and seropositivity to Trypanosoma cruzi within a rural community in Northeast Brazil. Am Heart J 1983; 105: 287-94.

20. Kennedy HL, Whitlock JA, Sprague MK, Kennedy LJ, Buckingham TA, Goldberg RJ - Long-term follow-up of asymptomatic health subjects with frequent and complex ventricular ectopy. N Engl J Med 1985; 312: 193-7.

21. Brodsky M, Wu D, Denes P, Kanakis C, Rosen KM - Arrhythmias documented by 24-hour continuous electrocardiographic monitoring in 50 male medical students without apparent heart disease. Am J Cardiol 1977; 39: 390-5.

22. Carrasco HA, Guerrero L, Parada H, Molina C, Vegas E, Chuecos R - Ventricular 
arrhythmias and left ventricular myocardium function in chronic chagasic patients. Int J Cardiol 1990; 28: 35-41.

23. Coura JR, Anunziato N, Willcox HP - Morbidade da doença de Chagas. I. Estudo de casos procedentes de vários estados do Brasil, observados no Rio de Janeiro. Mem Inst Oswaldo Cruz 1983; 78: 363-72.

24. Sobotka PA, Mayer JH, Bauernfeind RA, Kanakis C, Rosen KM - Arrhythmias documented by 24-hour continuous ambulatory electrocardiographic monitoring in young women without apparent heart disease. Am Heart J 1981; 101: 753-9.

25. Kennedy HL, Underhill SJ - Frequent or complex ventricular ectopy in apparently healthy subjects. A clinical study of 25 cases. Am J Cardiol 1976; 38: 141-8.

26. Grupi CJ, Sosa E, Carvalho JF, Antonelli RH, Bellotti G, Pileggi F - Variabilidade espontânea da extrassistolia ventricular na cardiopatia chagásica crônica. Arq Bras Cardiol 1991; 56: 445-50.

27. Brasil A - A mutabilidade electrocardiográfica na cardiopatia crônica chagásica. Rev Assoc Med Minas Gerais 1953; 4: 149-52

28. Pereira-Barretto AC, Bellotti G, Sosa E et al - Arritmias e a forma indeterminada da doença de Chagas. Arq Bras Cardiol 1986; 47: 197-9.

29. Pereira JB, Coura JR - Morbidade da doença de Chagas. Estudo seccional em uma área endêmica, Virgem da Lapa, MG. Rev Soc Bras Med Trop 1986; 19: 139-48.

30. Hiss RG, Lamb LE - Electrocardiographic findings in 122,043 individuals. Circulation 1962; 25: 947-61.
31. Goldsmith RS, Zárate RJ, Zárate LG, Kafan I, Jacobson LB, Morales G - Estudios clinicos y epidemiológicos de la enfermedad de Chagas en Oaxaca, Mexico, y un estudio complementário de 7 años. 1. Cerro del Aire. Bol Of Sanit Panam 1986; 100: 145-69.

32. Porto CC - O eletrocardiograma no prognóstico e evolução da doença de Chagas. Arq Bras Cardiol 1964; 17: 313-46.

33. Dias JCP, Kloetzel K - The prognostic value of the eletrocardiographic features of chronic Chagas’ disease. Rev Inst Med Trop São Paulo 1968; 10: 158-62.

34. Mady C, Cardoso RHA, Pereira-Barretto AC, Luz PL, Bellotti G, Pileggi F Survival and predictors of survival in patients with congestive heart failure due to Chagas' cardiomyopathy. Circulation 1994; 90: 3098-102.

35. Pereira-Barretto AC, Bellotti G, Deperon SD et al - O valor do eletrocardiograma na avaliação da função miocárdica dos portadores de doença de Chagas. Arq Bras Cardiol 1989; 52: 69-73.

36. Guiguer Jr N, De Paola AAV - Fibrilação atrial em pacientes sem história de cardiopatia. Rev Soc Cardiol Est SP 1994; 4: 251-9.

37. Marin-Neto JA, Simões MV, Ayres-Neto EM et al - Studies of the coronary circulation in Chagas'heart disease. São Paulo Medical Jornal/RPM 1995; 113 826-34.

38. Almeida EA, Martin CF - Estudo clínico e necroscópico de 65 casos de chagásicos crônicos referente à pesquisa de aterosclerose coronariana e sua repercussão no miocárdio. Rev Soc Bras Med Trop 1992; 25(supl III): 87-8. 